Southern Illinois University Edwardsville SPARK

SIUE Faculty Research, Scholarship, and Creative Activity

Fall 8-3-2015

\title{
The Compensatory Role of Implementation Intentions for Young Adults with Low Working Memory Capacity
}

J Thadeus Meeks

Southern Illinois University Edwardsville, jmeeks@siue.edu

Margarida Pitães

Arizona State University, margaridapitaes27@gmail.com

Gene A. Brewer

Arizona State University, ugabrewer@gmail.com

Follow this and additional works at: http://spark.siue.edu/siue_fac

Part of the Cognitive Psychology Commons

\section{Recommended Citation}

Meeks, J Thadeus; Pitães, Margarida; and Brewer, Gene A., "The Compensatory Role of Implementation Intentions for Young Adults with Low Working Memory Capacity" (2015). SIUE Faculty Research, Scholarship, and Creative Activity. 11.

http://spark.siue.edu/siue_fac/11 


\section{Cover Page Footnote}

This is the peer reviewed version of the following article: Meeks, J. T., Pitães, M., and Brewer, G. A. (2015) The Compensatory Role of Implementation Intentions for Young Adults with Low Working Memory Capacity. Appl. Cognit. Psychol., doi: 10.1002/acp.3151, which has been published in final form at http://dx.doi.org/ 10.1002/acp.3151. This article may be used for non-commercial purposes in accordance with Wiley Terms and Conditions for Self-Archiving. 
The Compensatory Role of Implementation Intentions for Young Adults with Low Working Memory Capacity

J. Thadeus Meeks ${ }^{1}$, Margarida Pitães ${ }^{2}$, and Gene A. Brewer ${ }^{2}$

${ }^{1}$ Southern Illinois University Edwardsville

${ }^{2}$ Arizona State University

\section{Author Notes}

J. Thadeus Meeks, Department of Psychology, University of Southern Illinois,

Edwardsville, Margarida Pitães, Department of Psychology, Arizona State University, Gene A.

Brewer, Department of Psychology, Arizona State University.

We would like to thank Molly Magary and Michael Brodsky for their help in collecting the data.

Correspondence concerning this article should be addressed to J. Thadeus Meeks, Department Psychology, University of Southern Illinois Edwardsville, 62026

E-mail: jmeeks@ siue.edu. Phone: (618) 650-3659. 


\begin{abstract}
Many factors improve prospective memory performance both inside and outside of the laboratory, including the detailed planning of the situational cue and intended action (i.e., implementation intentions). In the current study, we obtained measures of working memory capacity and laboratory event-based prospective memory performance in college-aged adults. Half of our participants formed an implementation intention in the prospective memory task. Due to evidence that implementation intentions increase the encoding/retrieval efficiency of the prospective memory, it was predicted that forming an implementation intention would serve as a compensatory strategy for those with low working memory ability. Our results supported this hypothesis in that working memory capacity no longer correlated with prospective memory performance when participants employed an implementation intention encoding strategy. These findings suggest that implementation intentions may be an effective way for individuals with low working memory capacity to improve their performance in an attentionaly demanding prospective memory task.
\end{abstract}

Keywords: event-based prospective memory, implementation intentions, working memory capacity 
The Compensatory Role of Implementation Intentions for Young Adults with Low Working

$$
\text { Memory Capacity }
$$

On a day-to-day basis, individuals establish many intentions that cannot be immediately accomplished due to various contextual, physical, or temporal constraints. Therefore, the ability to remember to perform an action at either the occurrence of a certain event (i.e., event-based prospective memory) or time (i.e., time-based prospective memory) is an important factor for successful living (Einstein \& McDaniel, 1990). Commonly cited examples of prospective memory in daily life include remembering to deliver an important message to a colleague, take a medication as prescribed, or attend a healthcare appointment. Depending on task demands and individual differences, the retrieval of deferred intentions vary on the extent to which capacitydemanding (versus more spontaneous) processes are necessary to be engaged (Einstein et al., 2005). For example, while the intention to deliver one message to a colleague is likely to be spontaneously retrieved, a person may be more likely to strategically monitor the environment if multiple cues or target actions are involved (e.g., if a person needs to remember to deliver the message to multiple colleagues and also make an important phone-call at some subsequent period of time; Cohen et al., 2008; Kliegel et al., 2000). Although there is a general bias to rely on a system that allows spontaneous prospective memory retrieval (Einstein et al., 2005), some specific demanding situations necessitate a more controlled approach to prospective remembering. Such prospective memory tasks could include those with multiple target events/actions, as opposed to a single target (Cohen et al., 2008; Einstein et al., 2005; Kliegel et al., 2000), highly important prospective memory tasks (Kliegel, Martin, McDaniel, \& Einstein, 2004), and prospective memory tasks where the target cues are nonfocal (Einstein et al., 2005). Considering that highly demanding intentions have been found to increase an individual's 
vulnerability to prospective failures in laboratory (Cohen et al., 2008) and in everyday life situations (e.g., workplace, medication, aviation; e.g., Dismukes, 2012), identifying ways to enhance performance in such tasks seems to be especially important.

Because of the prevalence and importance of completing intentions in a timely manner, much research has explored strategies that can improve prospective memory performance. One such strategy is the formation of an implementation intention that involves a detailed "if/whenthen" encoding plan that strengthens the association between the exact situation one will be in when a prospective cue is encountered and the target action to be performed in response to that cue (Gollwitzer, 1999). For example, as a way to increase the likelihood of remembering to take a medication, one may specify precisely where and when to take his or her medication as opposed to forming a less specific intention to take the mediation on a daily basis.

In more naturalistic settings, studies have shown that implementation intentions significantly promote adherence to a health behavior and specific treatment routines (e.g., blood glucose monitoring, Liu \& Park, 2004, compliance to a low fat-diet, Adriaanse, Vinkers, De Ridder, Hox, \& De Wit, 2011; Armitage, 2004, attend cervical cancer screening, Sheeran \& Orbell, 2000) and facilitate people's performance on important self-care tasks (Varley, Webb, \& Sheeran, 2011). Implementation intentions are also effective encoding strategies in laboratory settings, such as improving both younger and older people's prospective memory performance (e.g., McDaniel, Howard, \& Butler, 2008; McFarland \& Glisky, 2012; Meeks \& Marsh, 2010, but see Burkard, Rochat, Van der Linden, Gold, \& Van der Linden, 2014; McDaniel \& Scullin, 2010). Zimmermann and Meier (2010) found that implementation intentions especially enhanced prospective memory performance in older adults as compared to young adults and adolescents, corroborating a series of studies suggesting that individuals with reduced cognitive 
abilities (e.g., working memory capacity) may benefit more from the use of implementation intentions (patients with multiple sclerosis, Kardiasmenos et al., 2008, patients with schizotypal personality features, Chen et al., 2013). Along these lines, a body of research shows that younger adults with low working memory capacity often show poorer prospective memory performance when compared with high working memory capacity individuals (Brewer, Knight, Marsh, \& Unsworth, 2010; Smith \& Bayen, 2005). Given that we can reliably predict whether young adults have the propensity to commit prospective memory errors, it is important to investigate strategies that will improve prospective memory performance for those individuals with lower working memory abilities.

The dual-component model of working memory suggested by Unsworth and Engle (2007) serves as a useful theoretical framework for interpreting individual differences in prospective memory performance. This model proposes that active maintenance in primary memory and controlled retrieval from secondary memory jointly contribute to individual differences in working memory performance (e.g., Unsworth, Brewer, \& Spillers, 2011; Unsworth \& Spillers, 2010). To be successful in prospective memory, participants have to divide their attentional focus between the prospective memory and ongoing tasks, and they must also engage in controlled retrieval of the target action whenever a new cue is encountered (Brewer et al., 2010; Smith \& Bayen, 2005). Therefore, the dual component model suggests that individuals with high working memory capacity should make fewer prospective memory errors because they are better able to keep representations active in the focus of attention and/or are better at retrieving the appropriate target action (Marsh, Hicks, Cook, Hansen, \& Pallos, 2003: Unsworth \& Engle, 2008). These benefits could result from better encoding of the cue, the target, and the 
cue-target association, all of which are central to the proposed benefits of implementation intentions (e.g., McDaniel \& Scullin, 2010).

There are several hypotheses for why implementation intentions improve event-based prospective memory performance in general and differentially for individuals varying in working-memory capacity. Some propose that the use of an implementation intention automatizes the intention (i.e., the cue is at a heightened sensitivity and automatically elicits the associated action; Cohen \& Gollwitzer, 2008; Gollwitzer, 1999; Gollwitzer \& Sheeran, 2006). There is evidence, however, that implementation intentions do not actually automate the prospective response, but simply make the association between the cue and target stronger (McDaniel \& Scullin, 2010). This stronger association is a result of stronger cue-target encoding which thus makes noticing of the cue and/or the retrieval/execution of the intended action more reflexive, but not automatic (McDaniel \& Scullin, 2010). Along the same lines, Rummel, Einstein, and Rampey (2012) found that implementation intentions increased the spontaneous retrieval of an intention, presumably due to the heightened sensitivity to environmental cues and subsequent retrieval of the target action. If implementation intentions increase the reflexivity with which the intended action is triggered by the presence of the cue, then one might expect to see enhanced prospective memory performance, especially among individuals possessing low working memory capacity. In other words, the use of an implementation intention may lessen the amount of attentional resources needed to complete the intention for these individuals.

\section{The Current Study}

In the current study, we obtained measures of working memory capacity and event-based prospective memory performance in college-aged adults. The majority of the existing laboratory paradigms have focused mainly on prospective memory tasks that involve a limited amount of 
cues that are typically associated with one unique target action (e.g., a key press). Although such simple paradigms contribute to a better understanding of prospective memory, they do not completely capture many everyday complex situations where several intentions are planned to be executed at a later point in time. Therefore, in a laboratory paradigm utilizing multiple cue-target pairings, half of the participants in our study formed an implementation intention while the other half was given a typical event-based laboratory intention. Based on previous research that has shown the unique efficacy of implementation intentions in individuals with reduced controlled processes (Brom et al., 2013; Zimmerman \& Meier, 2010; but see Burkard et al., 2014), it was hypothesized that implementation intentions may serve as a compensatory encoding strategy that will equate performance across all levels of working memory capacity.

As a consequence of the less guidance provided in standard intentions encoding, studies suggest that a greater proportion of participants do not spontaneously form an effective encoding of the cue/target, as compared with implementation intention instructions (Cohen \& Gollwitzer, 2008; Kliegel et al., 2007). Research showing poorer cued recall performance in individuals with low working memory ability suggests that such individuals have a decreased ability both to select the appropriate encoding strategies and to use contextual cues/probes during retrieval, when compared to those with high working memory abilities (Unsworth, 2009). Therefore, we expected that the magnitude of improved performance from standard to implementation intention encoding instructions might be greater for individuals with lower working memory capacity, as they should be less likely to maintain a strong and unique association between the cue and target intention under standard instructions than the high working memory capacity group. Such improvement in prospective memory performance for individuals with low working memory ability seems to be expected, and especially important, in highly demanding tasks, given that no 
disproportionate working memory-related decline is found with less demanding prospective memory tasks (Brewer et al., 2010; Kliegel et al., 2000). In essence, implementation intentions may create a stronger, more reflexive association between the cue and target action (McDaniel \& Scullin, 2010; Rummel et al., 2012) and thus temporarily compensate for the lack of attentional control and/or retrieval ability in low working memory capacity individuals. Note that this is not the same thing as stating that implementation intentions increase natural working memory capacity.

Despite the fact that high working memory individuals may be more likely to spontaneously create a stronger link between a cue and the associated intention under standard instructions (e.g., Brom et al., 2013), recent research has shown that some individuals with relatively high working memory/functioning ability may also benefit from implementation intentions (Buckard et al., 2014; McFarland \& Glisky, 2011). These studies, however, have tested older and not younger adults. Thus, the current study was undertaken to explicitly investigate whether younger individuals that vary in working memory abilities equally benefit from the use of implementation intention encoding strategies in a complex prospective memory task. If implementation intentions do act as a compensatory strategy for those with lower capacity, the natural extension is that they could be used to improve the fulfillment of everyday intentions for these individuals, especially those intentions that are attentionally demanding.

\section{Method}

\section{Participants}

There were a total of 100 undergraduate Psychology students from Southern Illinois University Edwardsville used in the current analyses. ${ }^{1}$ Each participant was tested individually in 
sessions that took approximately $60 \mathrm{~min}$ to complete. Participants were randomly assigned to either a no implementation $(n=50)$ or an implementation intention condition $(n=50)$.

\section{Materials and Procedure}

After informed consent, participants received the instructions for the ongoing and prospective memory task. The ongoing task was a lexical decision task (LDT) that has commonly been used in event-based laboratory prospective memory studies (e.g., Meeks \& Marsh, 2010). The LDT contained 210 trials. Of these trials, there were 105 non-words and 101 non-cue words. The words were chosen from the English Lexicon Project Web Site (Balota et al., 2007) and were controlled on basic lexical characteristics (e.g., word-length, frequency). The non-words were created by rearranging the letters of another set of words chosen from the same database. The words and non-words were randomly presented. There were also four words used as prospective memory cues (eraser, credit, hotel, and thread). Two of the words were paired with a high associate word (eraser-pencil, credit-card) while two were paired with a low associate word (hotel-glass, thread-book). The cue words occurred on trials 50, 100, 150, and 200.

Participants first received instructions for the LDT. They were told to press, as quickly and accurately as possible, a designated key (the "J" key) if the letter string formed a valid English word and to press another key (the "F" key) if it did not form a valid English word. They were also told to press the spacebar (with their thumbs) when a waiting message appeared between each trial, which moved the computer to the next trial. This procedure is similar to past, related research (e.g., Brewer et al., 2010) and allowed time for the participants to make their verbal, prospective memory response. After this, the participants were given the prospective memory instructions. They were told that in the context of the LDT, if they ever encountered 
one of four words, they were to stop doing the LDT task and say aloud that cue word and the associated target word during the waiting message that occurred between LDT trials. The participants were also told that if they recognized the cue word but could not recall the associated target, they could indicate this by saying the cue word and then stating that they do not remember the target word. The participants were not given the prospective memory word pairs at this point, but were told they would appear on the next screen. After the participants read the instructions on the computer screen, the experimenter explained the tasks in detailed manner and the participant had an opportunity to ask any questions. After the experimenter was confident that the participant understood the instructions, the screen was advanced and the four cue words were simultaneously displayed along with the four associated target words. The participants were instructed to learn the word pairs for the task they were given during the last set of instructions. After the participants were confident they knew the pairs, they were tested on their memory for them. If they remembered all four cue-target pairings, they were allowed to advance to the next task. If they did not remember all four, they were given the list again and allowed to study it for as long as they felt necessary and then were tested again. This study-test cycle continued until the participant was able to recall all of the cue-target pairings. ${ }^{2}$

For the participants in the no implementation intention condition, the computer was advanced to a blank screen and they immediately completed an unrelated questionnaire that took approximately $5 \mathrm{~min}$. For those in the implementation intention condition, they did not start the survey immediately. Instead, they read aloud "When I see the word (hotel, eraser, thread, credit) while making a word decision, I will stop doing the lexical decision task and call out (hotel-glass, eraser-pencil, thread-book, credit-card) to the experimenter during the waiting message." These participants said this sentence twice for each set of word 
pairs and then immediately began the same unrelated questionnaire. This is a standard laboratory implementation intention procedure (e.g., Cohen \& Gollwitzer, 2008). After the survey, both groups started the ongoing and prospective memory task with no further mention of the prospective memory task. At the end of the LDT, the participants were asked to recall all four of the prospective memory word pairs.

After the LDT, the participants completed the automated version of the Operation Span task (Aospan; Unsworth, Heitz, Schrock, \& Engle, 2005). This required the participant to verify math problems along with memorizing series of letters that appeared on the screen one at a time for $800 \mathrm{~ms}$. The participant saw one letter and then had to determine if a math equation was correct. After anywhere from three to seven letters, the participant had to recall the letters in the order that they appeared by clicking on the appropriate boxes marked on the computer. After the Aospan, the participants completed another unrelated questionnaire that took approximately 5 min. Following this questionnaire, the participants completed the automated version of the Reading Span task (Arspan). This task is similar to the Aospan with the exception of using semantic sense judgments on sentences instead of the verification of math problems. When the participants finished the Arspan, they were thanked and debriefed.

\section{Statistical Analyses}

Prospective memory performance was operationalized as the proportion of all four cues that were successfully detected and verbally paired with the correct target word. ${ }^{3}$ We also analyzed performance including those trials where the participant recognized the cue word but either did not know the target word or said the incorrect target word. These instances were rare ( $2.5 \%$ of the total cue trials) and the pattern of results were identical using this measure (the results are not reported). For LDT latency (in ms), as is common practice (e.g., Brewer, 2011; 
Brewer et al., 2010; Meeks \& Marsh, 2010), we only used correct word trials (accuracy was around $95 \%$ in both conditions) and we trimmed reaction time trials $2.5 S D$ above and below an individual participant's mean word latency. The score for the Aospan and Arspan task were calculated by summing the total number of letters recalled in the correct position across the entire task (out of 75). The measure of working memory capacity used in the primary analyses was a composite measure combining the Aospan and Arspan scores. Both scores were transformed into z-scores and then averaged together for each participant (see Brewer et al., 2010 for a similar procedure). We believe that using the entire range of working memory capacity is more representative of the population as compared to using an extreme group design (Conway et al., 2005).

We initially performed t-tests comparing the two intentions conditions on Aospan scores, Arspan scores, composite working memory capacity, prospective memory performance, word latencies, and the percentage of cue-target pairs recalled at the end of the task (out of four). To examine working memory capacity and the interaction between intention condition and working memory capacity, hierarchical linear regression analyses were used for two separate dependent measures (prospective memory performance and word latency). In both regression analyses, Step 1 included intention condition and the centered composite working memory capacity measure while Step 2 included the two-way interaction between intention condition and working memory capacity. To follow up on the regression analysis, correlations between working memory capacity, word latency, and prospective memory performance were computed (both across all participants and separated by intention condition). 


\section{Results}

\section{Group Comparisons}

Please see Table 1 for overall descriptive statistics as well as the descriptive statistics separated by intention condition. For all statistical analyses, a conventional alpha level of .05 was used. In terms of intention condition, the implementation intention group had higher prospective memory performance, $t(98)=2.24, p=.027, d=.45 .^{4}$ The two conditions did not statistically differ on any working memory measure, the cue-target word recall measure (it was near ceiling for both groups), or the word latency measure. In sum, we replicated the typical benefit of implementation intentions to prospective memory performance (Cohen \& Gollwitzer, 2008; McDaniel et al., 2008; McFarland \& Glisky, 2012). Additionally, working memory capacity was equated between participants in both conditions.

\section{Regression and Correlation Results}

Please see Tables 2 and 3 for the results of the hierarchical regression analyses on prospective memory performance and word latencies, including standardized beta coefficients, $R^{2}$ change values for both steps, and overall $R^{2}$ model values. There were no significant findings in the word latency analysis (lowest $p=.202$ ). Regarding the primary analysis on prospective memory performance, Step 1 was significant, $F(2,97)=3.30, p=.041$. Within this step, only the coefficient related to the intention condition variable reached significance, $t(98)=2.43, p=$ .017 , which corresponds to the group comparison in showing that those in the implementation intention condition have better overall performance than those that did not form an implementation intention. The coefficient associated with working memory capacity was not significant, $t(98)=1.24, p=.218$. More importantly, these comparisons were qualified by a 
significant interaction between intention condition and working memory capacity in Step 2, $F(3$, 96) $=4.85, p=.003$.

To follow up on this interaction, we computed correlations (see Table 3). Across participants in both conditions, the only significant correlation was between word latency and prospective memory performance indicating that slower word latencies were related to higher performance. There was no correlation between word latency and working memory capacity, which is consistent with the lack of differences found in an extreme working memory group paradigm (Brewer et al., 2010). Central to the current research question, there was no correlation between working memory capacity and prospective memory performance, which may seem inconsistent with past research (Brewer et al., 2010; Smith \& Bayen, 2005). Given that those past studies did not use implementation intentions, however, a more apt cross-study comparison would be obtained by examining that correlation in the no implementation intention condition. In that condition, the expected correlation was found such that working memory capacity was positively correlated with prospective memory performance. In the implementation intention condition, however, this same correlation was not significant (and was even slightly negative). To further illustrate the nature of this interaction, we separated the participants into the top and bottom tertiles based on their composite working memory capacity score. As can been seen in Figure 1, there is a clear separation between the low and high working memory capacity individuals in the no implementation intention condition in terms of their prospective memory performance. In the implementation intention condition, however, prospective memory performance was very similar for the high and low working memory capacity individuals. As can be seen in Figure 2 (word latency) and Figure 3 (number of pairs recalled at the end of the 
prospective memory task), there were no apparent differences/patterns when looking across the different tertiles for our other two primary dependent measures.

Even though reaction times to word trials are commonly used to assess ongoing task interference caused by the prospective memory task, it is also possible that participants took advantage of the self-paced waiting period to review or rehearse their intention. ${ }^{5}$ As can be seen in Table 1, there was no overall prospective memory conditional difference and the average reaction times (in $\mathrm{ms}$ ) were relatively fast. This analysis did not include cue trials and was based on untrimmed data (the pattern of results remained the same for trimmed reaction times). Even though the reaction times were very fast on average, it is possible that on a limited amount of trials, participants paused to review/rehearse the intention, thus showing some cost of the implementation intention. In order to determine whether working memory capacity was related to waiting message latency and whether this interacted with the prospective memory condition, we conducted a similar regression analyses as with prospective memory performance and word latency (see Table 2). In this analysis, only the interaction between intention condition and working memory capacity reached significance, $F(3,96)=2.84, p=.042$. Follow-up correlations (Table 3) show that in the no implementation intention condition, there was a nonsignificant positive correlation between working memory capacity and waiting latencies. In the implementation intention condition, however, there was a significant negative correlation. Looking at the tertile descriptives (Figure 4), the high capacity individuals had slower waiting latencies as compared to the low capacity individuals in the no implementation intention condition. This pattern reversed in the implementation intention condition. Based on these results, it is possible that the use of an implementation intention either sped up waiting message latencies for those with high working memory capacity and/or slowed them down for those with 
low capacity. Visual inspection of the descriptives in Figure 4 might suggest that the former is more likely. If the latter is true, however, this could be indicative of an increased intention review/rehearsal process for the low capacity individuals when given an implementation intention. These results should be interpreted with caution, as it is not clear what processes are occurring during the waiting message period, especially considering the speed of the waiting message latencies.

Lastly, we examined the percentage of participants (separated by condition) that had each specified amount of cue detection (i.e., $0 \%, 25 \%, 50 \%, 75 \%$, and 100\%) (see Figure 5). This descriptive data relates to the possibility that those in the no implementation intention condition simply did not understand the instructions, even though they were well-explained and not complex. While there were more $0 \%$ responders in the no implementation intention condition, there were also more participants in that condition that detected either detected $25 \%$ or $50 \%$ of the cues. To explore this possibility further, we analyzed prospective memory performance in the same regression analysis previously described omitting the 20 total participants that did not detect a single cue. In this analysis, the interaction between working memory ability and prospective memory condition produced the same pattern of data and was marginally significant $(p=.055)$. If the results were indeed the results of instructional misunderstanding, we do not believe the pattern of results would have remained the same. In addition, while we do not believe prospective memory was on ceiling in either condition, it is informative to see the range of responses in each condition. Figure 4 shows that, as expected, there were less $0 \%$ responders and more $100 \%$ responders in the implementation intention group. Even so, there were still many participants across the range of cue detection values. While range restriction may be a possible limitation, there was some variability of cue detection values across conditions. These 
results are at least some evidence against the limitation of range restriction and the lack of instructional understanding.

\section{Discussion}

While implementation intentions seem to be an effective strategy to improve prospective memory performance, the question of interest is whether this applies for all individuals or if some people specially profit from implementation intentions. Based on previous research that showed prospective memory deficits in younger individuals with low working memory capacity, we sought to explore whether individual differences in working memory capacity are related to the efficacy of implementation intentions in a prospective memory task. Our results indicated that working memory ability alone did not significantly predict event-based prospective memory performance and that the use of implementation intentions was the reason. The results from the no implementation intention group correspond with extant literature that shows that working memory ability only relates to prospective memory ability when the prospective memory task requires a high amount of attentional demand (Brewer et al., 2010). Other research has also examined how individual differences in working memory ability relate to more attentionaly demanding intentions (e.g., multiple delayed actions; Kliegel et al., 2000). Similarly, we believe our prospective memory task was more attentionaly demanding in that we used four unique cuetarget pairs (Cohen, Jaudas, \& Gollwitzer, 2008; Einstein et al., 2005; Kliegel et al., 2000). In this context, our results revealed that when participants were instructed to form an implementation intention, working memory ability was no longer related to prospective memory performance (i.e., implementation intentions equated performance for those across the range of working memory ability), suggesting that those that have relatively low working memory capacity can improve their use of event cues in the service of remembering to complete future 
intentions that are attentionally demanding.

Although past laboratory studies on implementation intentions have used multiple cues, these cues are typically associated with one simple target action. This methodological aspect makes it less likely that implementation intentions operate simply through better retrospective memory of the cues, the targets, and/or the cue-target pairs. In our study, however, each of the four cue words was paired with a unique word and two of those cue-target pairs were low associates (e.g., thread-book). Thus, it is more likely that increased retrospective memory could be a candidate as an explanation for our benefit of implementation intentions. There are aspects of our method/results, however, that counter this explanation. Even though there were four unique cue-target pairs, two were high associate pairs (e.g., credit-card). These cue-target pairs were learned to criterion before the experiment began and were recalled at a very high rate (near ceiling) at the end of the experiment and recall did not differ as a function of condition. We conducted a post-hoc analysis including only those participants that recalled all four of the cuetarget pairings at the end of the study and the pattern of results did not change. In sum, there is not strong evidence that it was simply increased retrospective memory that led to our effect. The possibility remains, however, that more efficient retrieval of the target actions may create higher prospective memory performance in the presence of a distracting ongoing task. Thus, although the cues, targets, and cue-target associations were remembered equally, those with higher working memory capacity may have retrieved the target more quickly in the presence of a distracting ongoing task. The participants, however, were not under a time constraint to retrieve and report the target action once they noticed the cue. Participants were also given the opportunity to notify the experimenter if they detected a cue but did not remember the target word. As stated in the results, we analyzed prospective memory performance including these 
responses and those where the incorrect target word was spoken. These trials were rare and did not make a difference in the pattern of results.

Perhaps the more likely explanation of the current findings is that the implementation intention heightened sensitivity to the event cues, which thus increased the chances of noticing the cues and subsequently retrieving the intention (Cohen \& Gollwitzer, 2008; Gollwitzer, 1999; Gollwitzer \& Sheeran, 2006). Even though this explanation does not necessitate that this process is made automatic by the implementation intention (McDaniel \& Scullin, 2010), it does suggest that it can occur with less attentional focus, which may be even more important when multiple cues and targets are used (as compared to fewer cues and only one target action, e.g., McDaniel \& Scullin, 2010). Perhaps the increased sensitivity to the cues counters the lower attentional control ability of those with low working memory capacity and thus serves as a temporary compensatory mechanism. Unsworth and Engle (2007) proposed that the attentional control mechanism that is affected by working memory reflects the ability to maintain representations in primary memory amidst distraction. It is possible that implementation intentions could compensate for low control ability by keeping the cues more active in primary memory. Another possibility is that the cues/cue-target associations are not actively maintained, but the general goal (i.e., fulfill the intention) is (e.g., Cowan, 2005; Unsworth \& Engle, 2007). Regardless of whether it is the specific cues/targets or the goal that is active, the activation may not be in the focus of attention, but rather at a heightened sense of activation outside of the primary focus of attention (Cowan, 1997). This increased activation may help protect low working memory ability individuals from the distraction they face in the task, which would normally lead to an inability to notice the cue and retrieve the intention (e.g., the ongoing task, task unrelated thoughts). 
Although our results are consistent with recent research that found that only older adults with low cognitive ability profited from the formation of implementation intentions (Brom et al., 2013), Buckard and colleagues (2014) found that implementation intentions were only effective for older adults with high working memory ability. Although our results seem to be inconsistent with these results from aging populations, Buckard and colleagues tested older adults with reported memory deficits and claimed that one interpretation of these discrepant results is that the relationship between the efficacy of implementation intentions and cognitive resources is inversely U-shaped. Therefore, implementation intentions would not be very efficient in older individuals with very limited cognitive resources because some minimum cognitive ability is needed to utilize these encoding strategies. Even so, McFarland and Glisky (2011) found that implementation intentions were equally effective for both high and low frontal lobe functioning older adults and that the high functioning group had better prospective memory regardless of whether an implementation intention was used. Future research is needed to examine whether these inconsistencies are due to the samples tested and/or some other methodological factor.

Aside from the possible limitations of range restriction and instructional understanding addressed in the Results section, a few other limitations of this study need to be considered. Although prospective memory performance was not on ceiling, it was still over 60 percent overall. Perhaps with an even more demanding task, those with high working memory capacity might benefit from an implementation intention. Originally, the study was designed to include attentional demand as a factor by using high and low-associate target pairs (McDaniel et al., 2004, Experiment 2), but performance did not differ for these two cue-target types and the pattern of findings was not dependent upon this factor. This may be due to the fact that because four cue-target pairs were given in a within-subject manipulation, the entire intention became 
attentionally demanding (Cohen et al., 2008). Previous manipulations of this type utilized fewer cue-target pairs in a between-subject manner (McDaniel et al., 2004, Experiment 2). Perhaps in a more direct, between-subject manipulation using an even more demanding intention, high working memory ability participants would show a benefit from implementation intentions. A similar manipulation would also generally help determine whether the compensatory effects would differ at varying degrees of attentional demand. As already mentioned, we believe that our prospective memory task was attentionally demanding, and thus may have required some level of monitoring (Einstein et al., 2005). Although many event-based intentions can be achieved using spontaneous retrieval, there is evidence that, with more complex intentions, people need to devote attention to noticing an event cue/s (e.g., remembering turn a coffee pot off if you typically do not make coffee, delivering multiple messages). Our results suggest that those with lower working memory ability can use implementation intentions to improve their performance on such intentions, perhaps by reducing the amount of attentional resources needed to detect the cue. In many instances, however, little (if any) attentional resources are needed to detect event cues, thus eliciting more of a spontaneous retrieval of the intention (e.g., remembering to turn the coffee pot off if it is a daily task, delivering one message). Future research should continue to explore the implementation intention compensation effect in these different situations.

Another limitation is that the participants in the implementation intention condition received more exposure to the cue-target pairings before the onset of the task as compared to the standard instruction condition. While time was likely not a primary factor (it does not take long to repeat eight sentences), it could be argued that it was the additional exposure to the cues in general led to our results and not the specific implementation intention itself. From an applied perspective, the extra exposure is a natural component of forming implementation intentions and 
our research suggests that the additional time/exposure may only be justified for those with low working memory capacity. It is possible that other time-consuming strategies (e.g., simple repetition) would not be as effective as an implementation intention. These practices may be more detrimental than using no strategy as they could be both resource and time-consuming. From both an applied and theoretical perspective, research should continue to isolate the roles time and exposure have in these prospective memory encoding effects. This could be achieved by including a control condition that was equated to the implementation intention in terms of time and exposure, but lacked the essential nature of the implementation intention. In a related sense, a last limitation concerns our lack of a no-intention control group. While this omission does not challenge the fact that prospective memory differences exist, it does limit the theoretical interpretation of that data. Research suggests that longer reaction times during an ongoing task are often indicative of allocating attention to the monitoring of event cues at a cost to the ongoing task (e.g., Brewer, 2011; Smith \& Bayen, 2004). Our results showed no differences in any word latency measure. The lack of difference in this measure between low and high working memory individuals is consistent with past research (Brewer et al., 2010). This is also at least some evidence that the compensatory effect of implementation intentions were not a result of the low working memory individuals perceiving the prospective memory task as more important and thus devoting more attention to the intention and away from the ongoing task. Without a control group to provide baseline reaction times, however, it is more difficult to make firm conclusions about the role of monitoring in our effects. Future work should consider this limitation. In addition, it is possible that the low working memory capacity individuals increased their review/rehearsal of the intention during the waiting message when given an implementation intention. As noted in the Results, this should be interpreted with caution. Even if the results do 
indicate that this is occurring, this small cost does not necessarily diminish the positive benefits of implementation intentions for those with low capacity.

Due to evidence that implementation intentions are effective both in the laboratory and in naturalistic settings, it is tempting to conclude that these results should apply outside of the laboratory. There are, however, potential fundamental differences between our laboratory intention and many everyday intentions. For example, the delay between intention formation and realization may differ in these two contexts. Given that the time between intention formation and completion in our study was not very long (about $15 \mathrm{~min}$ ), it would be advantageous to know if these effects occur in more delayed intentions that often occur in our lives. Other differences could include the number and types of cues and target actions as well as the nature of the ongoing tasks. We do feel that while simple intentions are prevalent in daily life, our inclusion of multiple cues and distinct target actions (as compared to similar research) more closely replicates complex intentions where errors could become more costly (e.g., workplace settings, medicine, aviation). Lastly, it would be useful to know how much awareness those with low working memory capacity have about their prospective memory ability. In general, individuals are at least somewhat aware of their own prospective memory ability (e.g., Meeks, Hicks, \& Marsh, 2007). If individuals with lower working memory ability are not aware, it may not be clear to them that any strategy is needed to improve their prospective memory performance. Regardless, our results are preliminary evidence that implementation intentions do serve a compensatory role for younger adults with lower working memory ability. 


\section{References}

Adriaanse, M. A., Vinkers, C. D., De Ridder, D. T., Hox, J. J., \& De Wit, J. B. (2011). Do implementation intentions help to eat a healthy diet? A systematic review and metaanalysis of the empirical evidence. Appetite, 56, 183-193.

Armitage, C. J. (2004). Evidence that implementation intentions reduce dietary fat intake: A randomized trial. Health Psychology, 23, 319-323.

Balota, D. A., Yap, M. J., Cortese, M. J., Hutchison, K. A., Kessler, B., Loftis, B., Neely, J. H., Nelson, D. L., Simpson, G. B., \& Treiman, R. (2007). The English Lexicon Project. Behavior Research Methods, 39, 445-459.

Brewer, G. A. (2011). Analyzing response time distributions. Zeitschrift für Psychologie, 219, 117-124.

Brewer, G. A., Knight, J. B., Marsh, R. L., \& Unsworth, N. (2010). Individual differences in event-based prospective memory: Evidence for multiple processes supporting cue detection. Memory \& Cognition, 38, 304-311.

Brom, S. S., Schnitzspahn, K. M., Melzer, M., Hagner, F., Bernhard, A., \& Kliegel, M. (2013). Fluid mechanics moderate the effect of implementation intentions on a health prospective memory task in older adults. European Journal of Ageing, 11, 1-10.

Burkard, C., Rochat, L., Van der Linden, A. C., Gold, G., \& Van der Linden, M. (2014). Is working memory necessary for implementation intentions to enhance prospective memory in older adults with cognitive problems? Journal of Applied Research in Memory and Cognition, 3, 37-43. 
Chen, X. J., Wang, Y., Liu, L. L., Shi, H. S., Wang, J., Cui, J. F., ... \& Chan, R. C. (2013). The effect and mechanisms of implementation intentions on prospective memory in individuals with and without schizotypal personality features. Memory, 22, 1-11.

Cohen, A. L., \& Gollwitzer, P. M. (2008). The cost of remembering to remember: Cognitive load and implementation intentions influence ongoing task performance. In M. Kliegel, M. A. McDaniel, \& G. O. Einstein (Eds.), Prospective memory: Cognitive, neuroscience, developmental, and applied perspectives (pp. 367-390). Mahwah, NJ: Erlbaum.

Cohen, A. L., Jaudas, A., \& Gollwitzer, P. M. (2008). Number of cues influences the cost of remembering to remember. Memory \& Cognition, 36, 149-156.

Conway, A. R., Kane, M. J., Bunting, M. F., Hambrick, D. Z., Wilhelm, O., \& Engle, R. W. (2005). Working memory span tasks: A methodological review and user's guide. Psychonomic Bulletin \& Review, 12, 769-786.

Cowan, N. (1997). Attention and memory. New York, NY: Oxford University Press.

Cowan, N. (2005). Working memory capacity. New York, NY: Psychology Press.

Dismukes, R. K. (2012). Prospective memory in workplace and everyday situations. Current Directions in Psychological Science, 21, 215-220.

Einstein, G. O., \& McDaniel, M. A. (1990). Normal aging and prospective memory. Journal of Experimental Psychology: Learning, Memory, and Cognition, 16, 717-726.

Einstein, G. O., McDaniel, M. A., Thomas, R., Mayfield, S., Shank, H., Morrisette, N., \& Breneiser, J. (2005). Multiple processes in prospective memory retrieval: Factors determining monitoring versus spontaneous retrieval. Journal of Experimental Psychology: General, 13, 327-342. 
Gollwitzer, P. M. (1999). Implementation intentions: Strong effects of simple plans. American Psychologist, 54, 493-503.

Gollwitzer, P. M., \& Sheeran, P. (2006). Implementation intentions and goal achievement: A meta-analysis of effects and processes. Advances in Experimental Social Psychology, 38, 69-119.

Kardiasmenos, K. S., Clawson, D. M., Wilken, J. A., \& Wallin, M. T. (2008). Prospective memory and the efficacy of a memory strategy in multiple sclerosis. Neuropsychology, 22, $746-754$.

Kliegel, M., Martin, M., McDaniel, M., \& Einstein, G. (2004). Importance effects on performance in event-based prospective memory tasks. Memory, 12, 553-561.

Kliegel, M., Martin, M., McDaniel, M. A., Einstein, G. O., \& Moor, C. (2007). Realizing complex delayed intentions in young and old adults: The role of planning aids. Memory \& Cognition, 35, 1735-1746.

Kliegel, M., McDaniel, M. A., Einstein, G.O. (2000). Plan formation, retention, and execution in prospective memory: A new approach and age-related effects. Memory \& Cognition, 28, 1041-1049.

Liu, L. L., \& Park, D. C. (2004). Aging and medical adherence: The use of automatic processes to achieve effortful things. Psychology and Aging, 19, 318-325.

Marsh, R. L., Hicks, J. L., Cook, G. I., Hansen, J.S., \& Pallos, A. L. (2003). Interference to ongoing activities covaries with contextual associations of fulfilling them. Journal of Experimental Psychology: Learning, Memory, and Cognition, 29, 861-870.

McDaniel, M. A., Guynn, M. J., Einstein, G. O., \& Breneiser, J. (2004). Cue-focused and 
reflexive-associative processes in prospective memory retrieval. Journal of Experimental Psychology: Learning, Memory, and Cognition, 30, 605-614.

McDaniel, M. A., Howard, D. C., \& Butler, K. M. (2008). Implementation intentions facilitate prospective memory under high attention demands. Memory \& Cognition, 36, 716-724.

McDaniel, M. A., \& Scullin, M. K. (2010). Implementation intention encoding does not automatize prospective memory responding. Memory \& Cognition, 38, 221-232.

McFarland, C. P., \& Glisky, E. L. (2011). Implementation intentions and prospective memory among older adults: An investigation of the role of frontal lobe function. Aging, Neuropsychology, and Cognition, 18, 633-652.

McFarland, C. P, \& Glisky, E. L. (2012). Implementation intentions and imagery: Individual and combined effects on prospective memory among young adults. Memory \& Cognition, 40, 62-69.

Meeks, J. T., Hicks, J. L., \& Marsh, R. L. (2007). Metacognitive awareness of event-based prospective memory. Consciousness and Cognition, 16, 997-1004.

Meeks, J. T., \& Marsh, R. L. (2010). Implementation intentions about nonfocal event-based prospective memory tasks. Psychological Research, 74, 82-89

Rummel, J., Einstein, G. O., \& Rampey, H. (2012). Implementation-intention encoding in a prospective memory task enhances spontaneous retrieval of intentions. Memory, 20, 803817.

Sheeran, P., \& Orbell, S. (2000). Using implementation intentions to increase attendance for cervical cancer screening. Health Psychology, 19, 283-289.

Smith, R. E., \& Bayen, U. J. (2004). A multinomial model of event-based prospective memory. Journal of Experimental Psychology: Learning, Memory, \& Cognition, 30, 756-777. 
Smith, R. E., \& Bayen, U. J. (2005). The effects of working memory resource availability on prospective memory: A formal modeling approach. Experimental Psychology, 52, 243256.

Unsworth, N. (2009). Examining variation in working memory capacity and retrieval in cued recall. Memory, 17, 386-396.

Unsworth, N., Brewer, G. A., \& Spillers, G. J. (2011). Variation in working memory capacity and forgetting over both the short and the long term: An application of the population dilution model. Journal of Cognitive Psychology, 23, 243-255.

Unsworth, N., \& Engle, R. W. (2007). The nature of individual differences in working memory capacity: Active maintenance in primary memory and controlled search from secondary memory. Psychological Review, 114, 104-132.

Unsworth, N., \& Engle, R. W. (2008). Speed and accuracy of accessing information in working memory: An individual differences investigation of focus switching. Journal of Experimental Psychology: Learning, Memory, and Cognition, 34, 616-630.

Unsworth, N., Heitz, R. P., Schrock, J. C., \& Engle, R. W. (2005). An automated version of the operation span task. Behavior Research Methods, 37, 498-505.

Unsworth, N., \& Spillers, G. J. (2010). Working memory capacity: Attention control, secondary memory, or both? A direct test of the dual-component model. Journal of Memory and Language, 62, 392-406.

Varley, R., Webb, T. L., \& Sheeran, P. (2011). Making self-help more helpful: A randomized controlled trial of the impact of augmenting self-help materials with implementation intentions on promoting the effective self-management of anxiety symptoms. Journal of Consulting and Clinical Psychology, 79, 123-128. 
Zimmermann T. D., \& Meier B. (2010). The effect of implementation intentions on prospective memory performance across the lifespan. Applied Cognitive Psychology, 24, 645-658. 


\section{Footnotes}

${ }^{1}$ There were originally 104 participants in the sample but we excluded four participants because they did not recall any of the four cue-target pairings after the prospective memory task. We analyzed the data with the subjects included, however, and there were no differences in the pattern of findings.

${ }^{2}$ Although we did not collect data on the number of participants that needed extra studytest cycles, the experimenters reported that most participants did not need more than one cycle. There were only four cue-target pairings and half of those were high associates. In this sense, given that we have a considerate sample size, even if group differences exist, we do not believe that the minimal amount of participants that needed extra time would account for differences in our results. In addition, there is no reason to believe that there would be a difference between the two prospective memory encoding groups as the manipulation occurred after the participants learned the cue-target pairs to criteria. Learning all cue-target pairings to criteria helped ensure that any group differences were not due to a failure of initial encoding, even though some participants may not have as reflexively retrieved the associations later when encountering the cue during the ongoing task (Unsworth, 2009)

${ }^{3}$ Late responses (defined here as any verbal response more than two trials after the cue word) were very rare and did not affect the results. Thus, we did not include them in the analyses. In addition, some participants began their verbal response before the waiting message. These occurrences were counted as correct. This element of the study, however, limited our ability to examine the word latencies on cue trials (i.e., cue interference). Even if participants did not begin the verbal response while the cue was on the screen, it is possible that they remained on the trial while they recalled the intention (before moving on to the waiting 
message). This, combined with the limited amount of cue detection trials, did not allow for meaningful cue interference analyses.

${ }^{4}$ The independent samples t-test did violate the assumption of homogeneity of variance.

The corrected results, however, produced identical results, $t(96.21)=2.24, p=.027$.

${ }^{5}$ We would like to thank Gil Einstein for suggesting this analysis. 
Table 1

Descriptive Statistics for all Measures as a Function of Intention Condition

\begin{tabular}{|c|c|c|c|}
\hline & $\begin{array}{c}\text { Overall }(N= \\
100)\end{array}$ & No II $(n=50)$ & II $(n=50)$ \\
\hline & Mean (SD) & $\operatorname{Mean}(S D)$ & Mean $(S D)$ \\
\hline Aospan Total & $56.65(13.43)$ & $58.30(13.30)$ & $55.00(13.49)$ \\
\hline Arspan Total & $51.50(14.29)$ & $54.04(12.85)$ & $48.96(15.30)$ \\
\hline WMC composite (centered) & $0.00(0.83)$ & $0.14(0.81)$ & $-0.14(0.83)$ \\
\hline PMP* & $0.63(0.39)$ & $0.55(0.40)$ & $0.72(0.35)$ \\
\hline Word Latency & $833.53(130.52)$ & $838.08(117.95)$ & $828.97(143.06)$ \\
\hline Pairs Recalled & $3.79(0.56)$ & $3.80(0.53)$ & $3.78(0.58)$ \\
\hline Waiting Message Latency & $331.62(137.17)$ & $346.19(141.49)$ & $317.04(132.52)$ \\
\hline
\end{tabular}


Table 2

Hierarchical Regression Results with II condition and WMC Predicting PMP

\begin{tabular}{|c|c|c|c|}
\hline Predictor Variables & PMP & Word Latency & Waiting Latency \\
\hline \multicolumn{4}{|l|}{ Step 1} \\
\hline II Condition & $0.24 *$ & -0.06 & -0.09 \\
\hline WMC & 0.12 & -0.13 & 0.001 \\
\hline$R^{2}$ Change & $0.06^{*}$ & 0.02 & 0.01 \\
\hline \multicolumn{4}{|l|}{ Step 2} \\
\hline II Condition x WMC & $-0.84 * *$ & -0.20 & $-0.88 * *$ \\
\hline$R^{2}$ Change & $0.07 * *$ & 0.004 & $0.07 * *$ \\
\hline Overall $R^{2}$ & 0.13 & 0.02 & 0.08 \\
\hline
\end{tabular}

Note: II = Implementation Intention; WMC = Centered Working Memory Composite Score; $\mathrm{PMP}=$ Prospective Memory Performance

Note: Standardized coefficients reported for ease of interpretation; ${ }^{*} p<0.05 ; * * p<0.01$ 
Table 3

Correlations between the Relevant Dependent Measures Pooling Over Both Conditions and for each Condition Separately

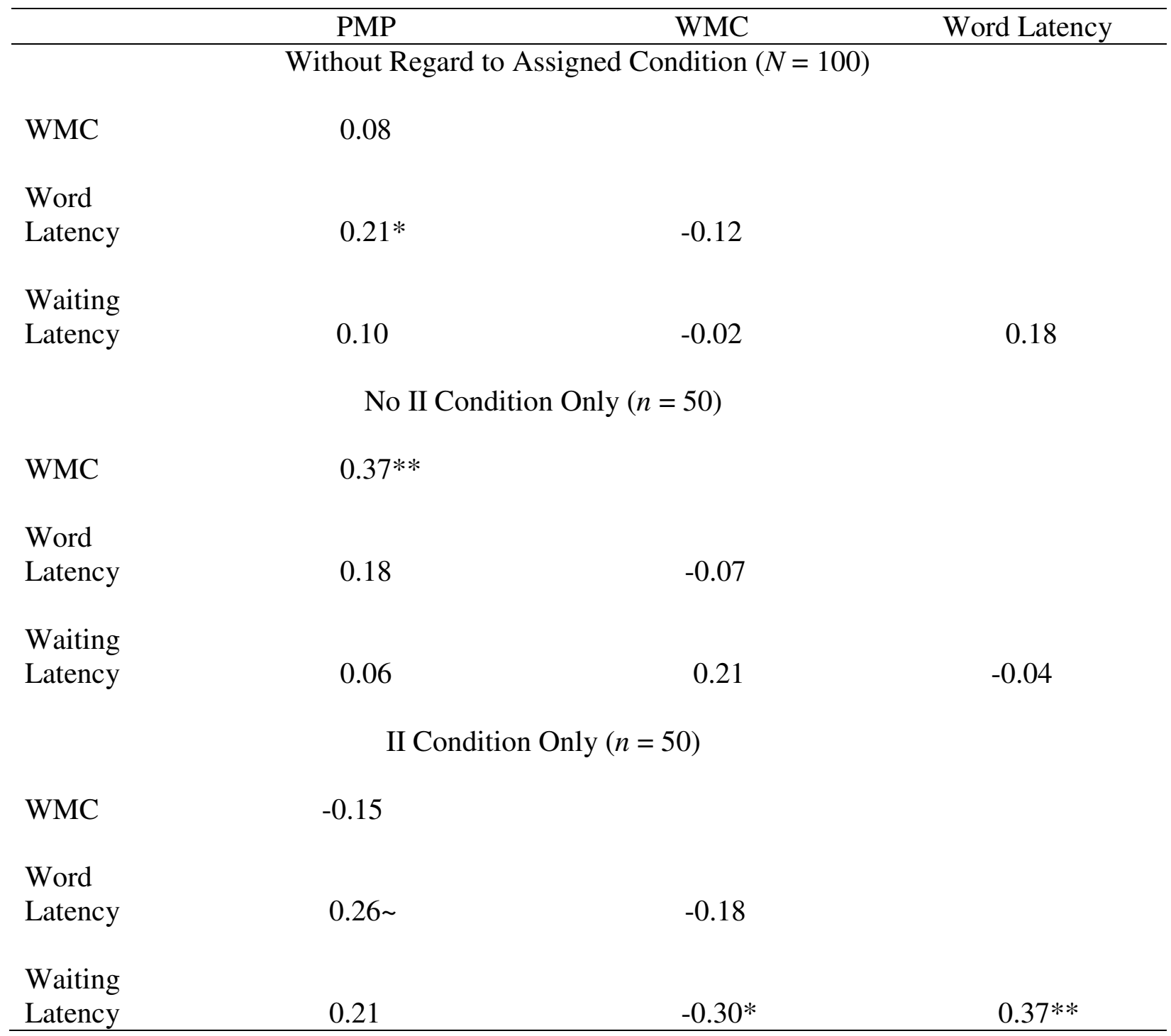

Note: II = Implementation Intention; WMC = Centered Working Memory Composite Score; PMP $=$ Prospective Memory Performance

$* p<0.05 ; * * p<0.01 ; \sim p=0.06$. 


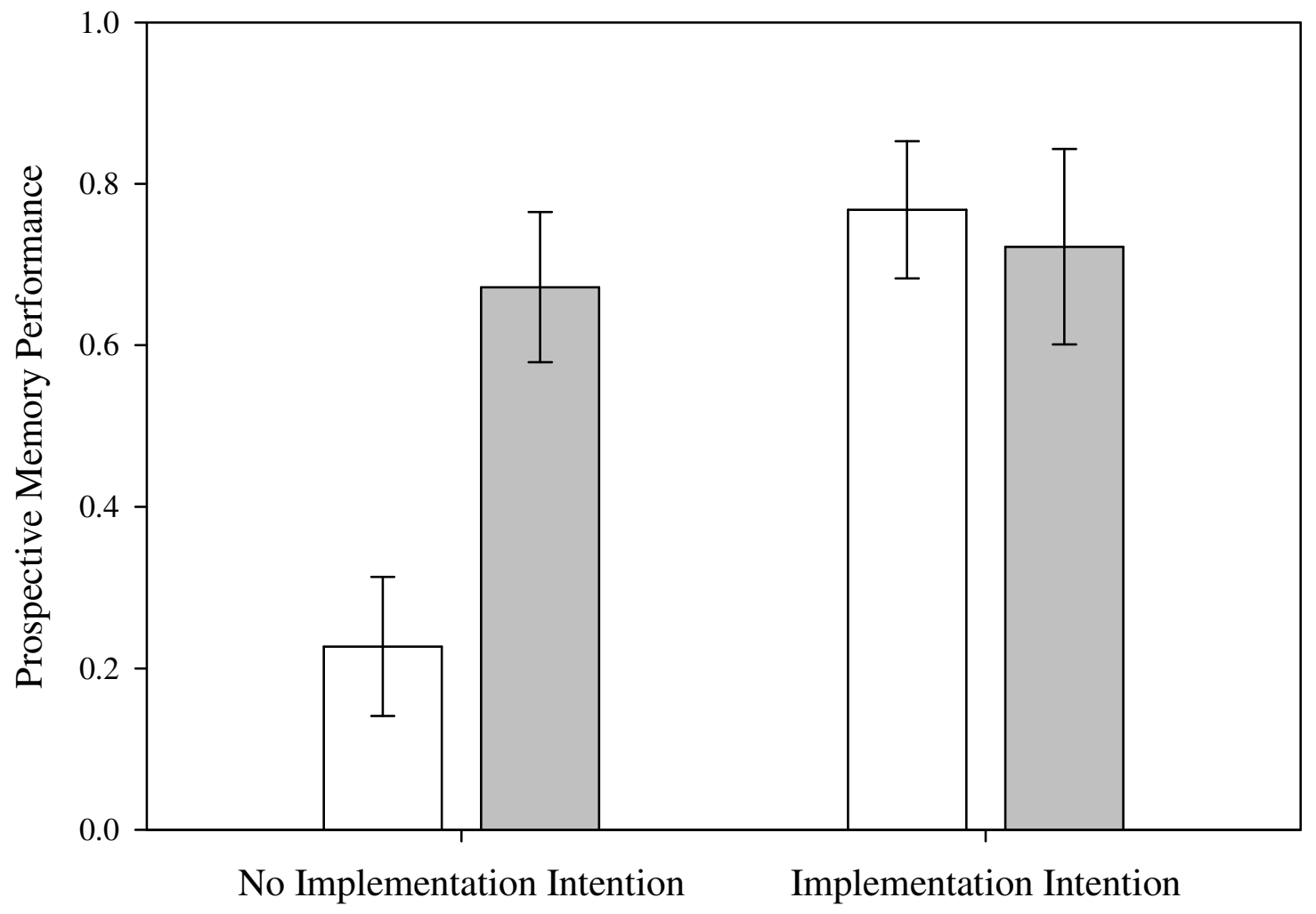

Figure 1. Prospective memory performance (bars represent standard errors) as a function of condition and $\mathrm{WMC}$ capacity (low $\mathrm{WMC}=$ bottom tertile, high $\mathrm{WMC}=$ top tertile). 


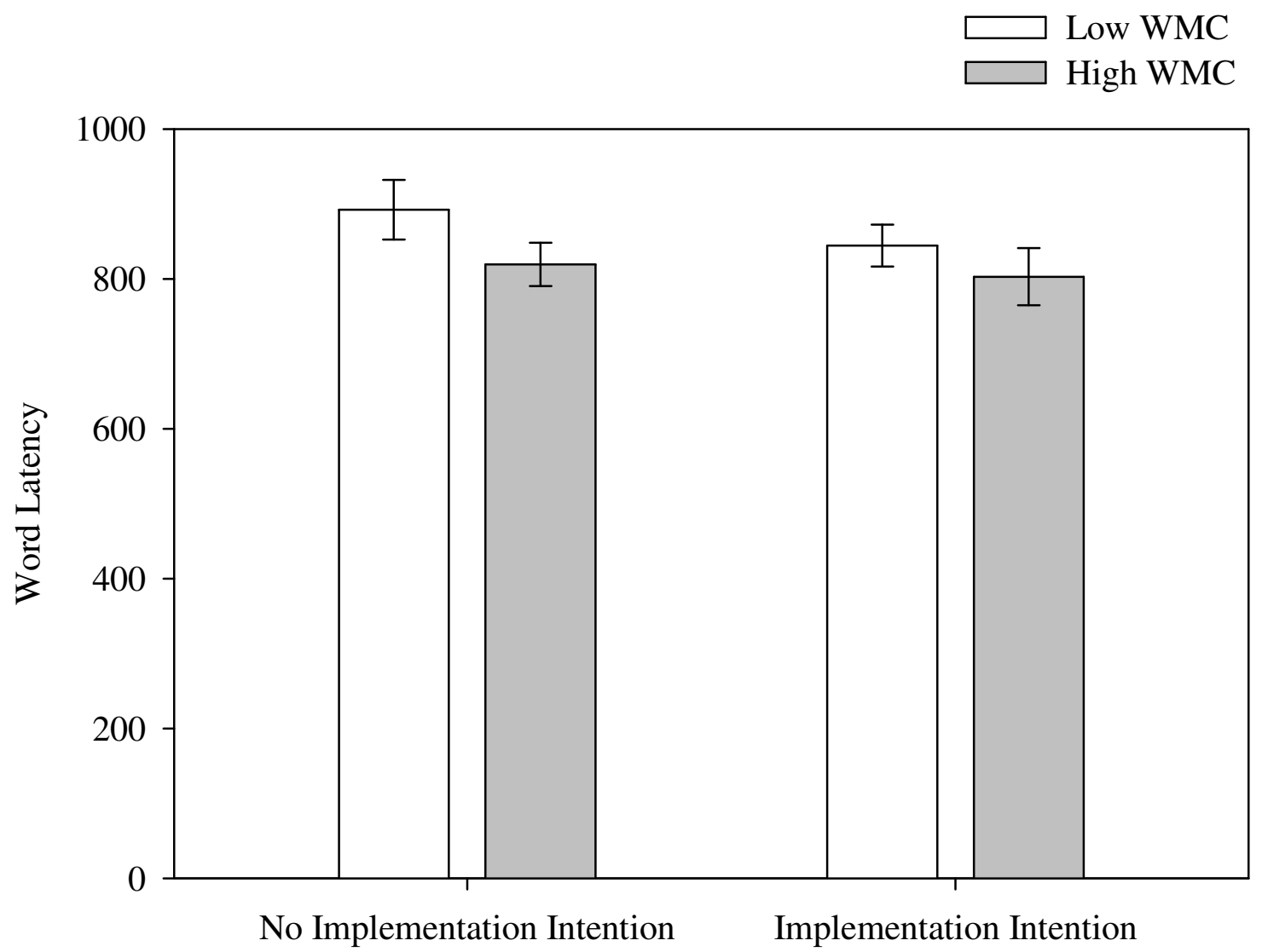

Figure 2. Word latency (in ms) as a function of condition and WMC capacity (low WMC = bottom tertile, high WMC = top tertile). The error bars represent the standard error of the mean. 


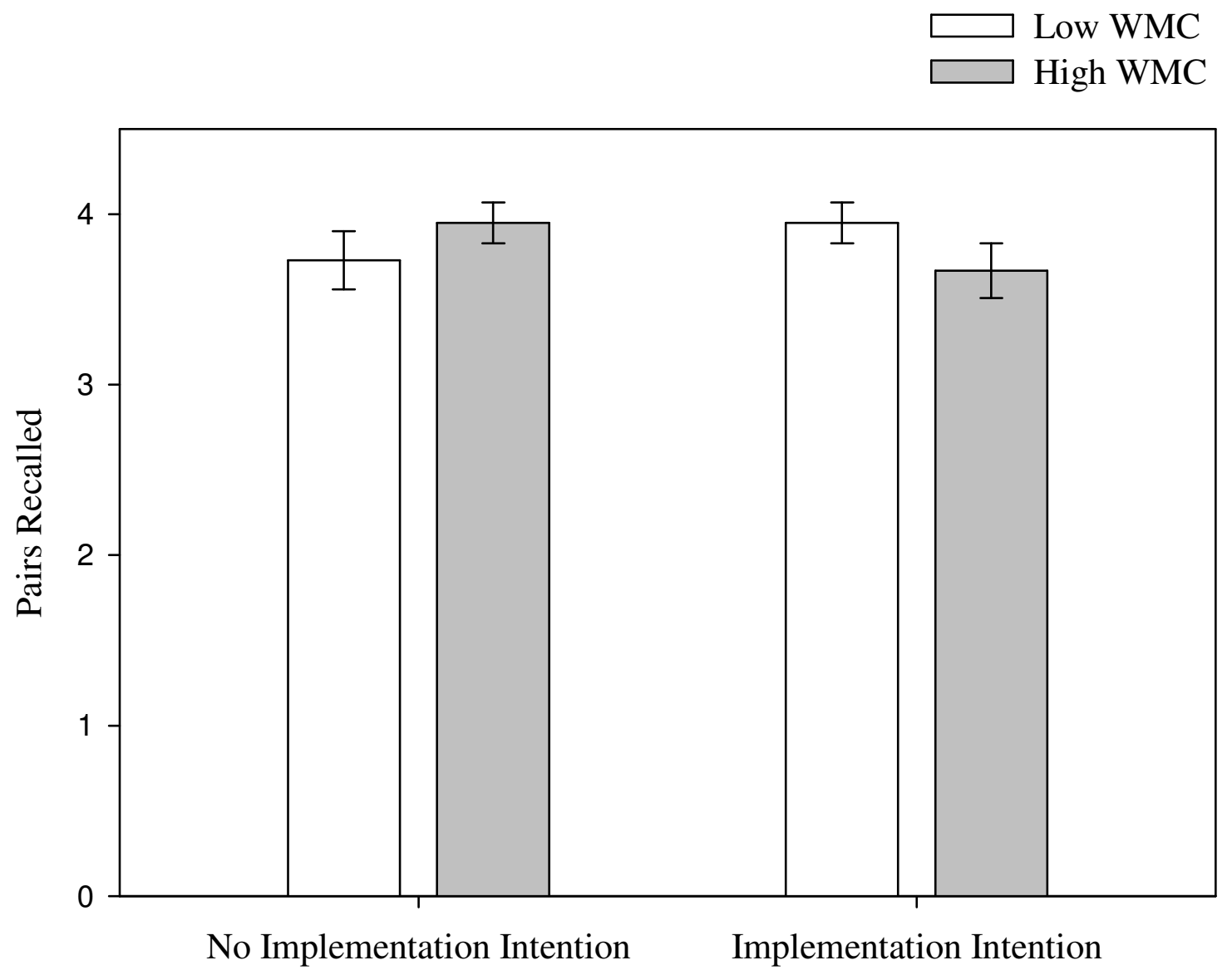

Figure 3. Pairs recalled (out of four) as a function of condition and WMC capacity (low WMC $=$ bottom tertile, high $\mathrm{WMC}=$ top tertile). The error bars represent the standard error of the mean. 


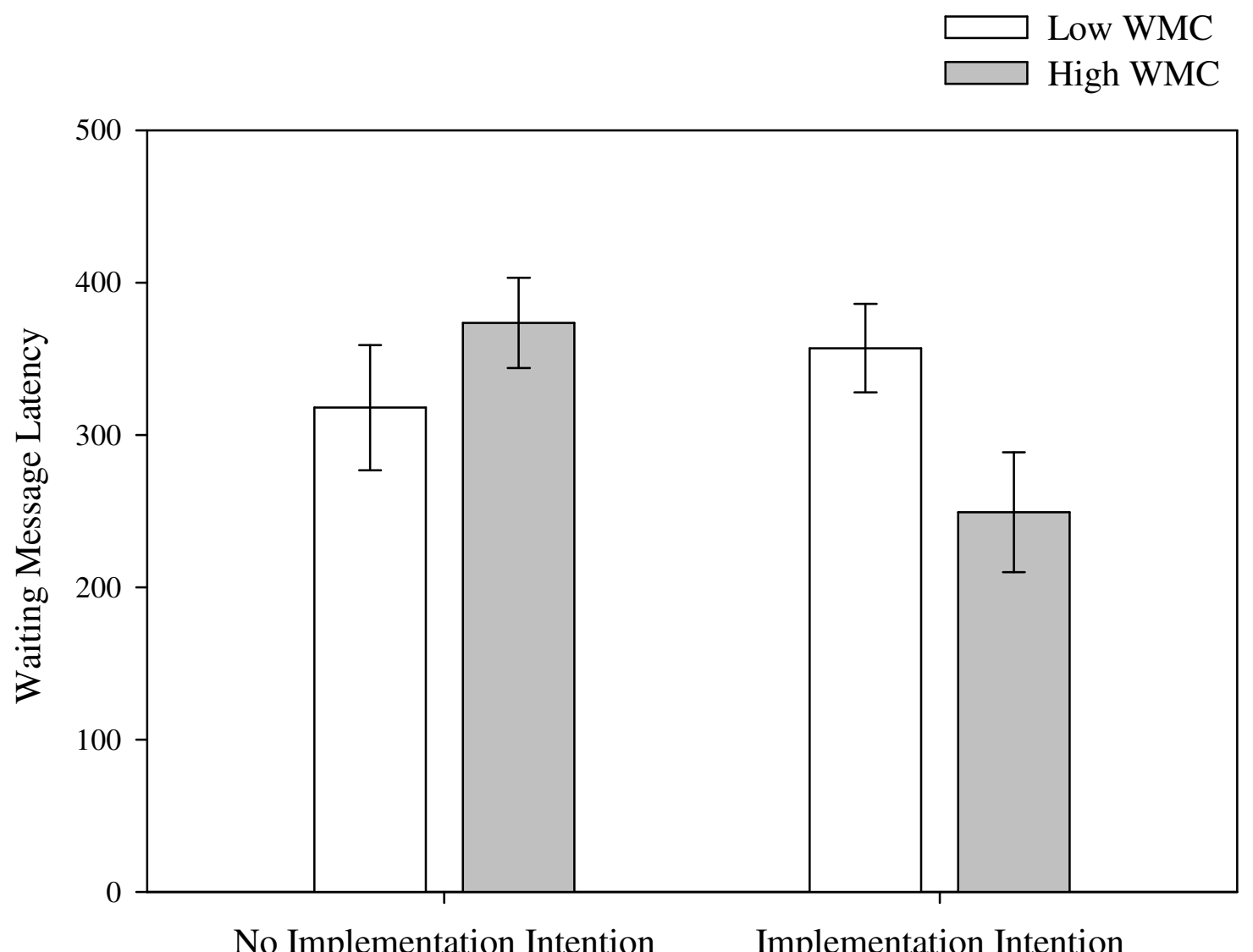

Figure 4. Waiting message latency (in ms) as a function of condition and WMC capacity (low $\mathrm{WMC}=$ bottom tertile, high $\mathrm{WMC}=$ top tertile $).$ The error bars represent the standard error of the mean. 
No Implementation Intention

Implementation Intention

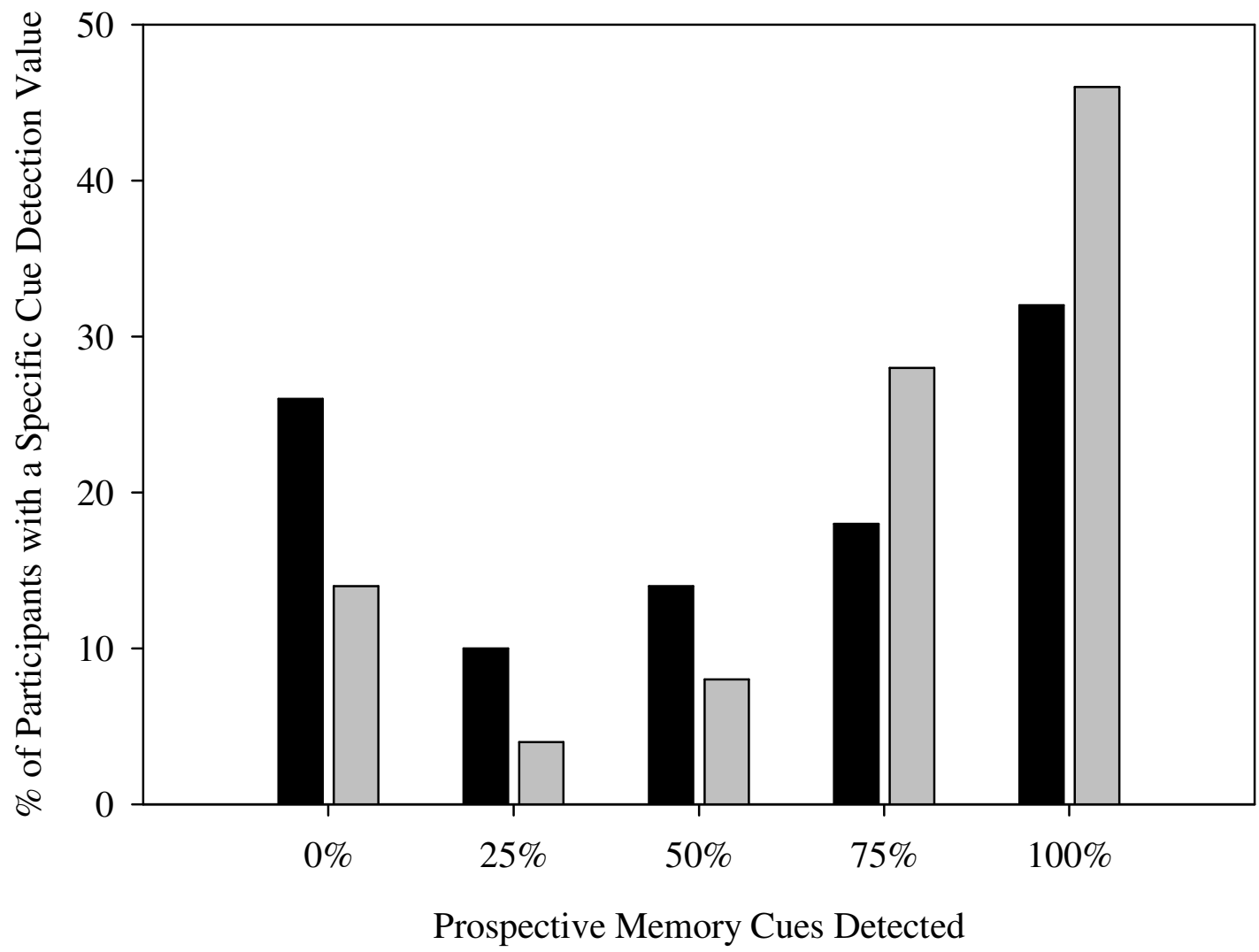

Figure 5. The percentage of participants in each condition that detected a specific amount of cues. 